\title{
Alter et Ego sont dans un bateau
}

\author{
Martin Winckler et Marc Zaffran
}

Dans La nuit sera calme (1974), Romain Gary, empruntant le nom de son ami François Bondy, répond en fait aux questions qu'il se pose à lui-même. Ici, Marc Zaffran s'entretient avec son pseudonyme littéraire, Martin Winckler.

Marc Zaffran : Comment veux-tu qu'on procède?

Martin Winckler : Je ne comprends pas ta question.

M. Z. : J'ai proposé à @nalyses une interview croisée, mais qui interroge qui ?

M. W. : Mmhhh... L'écrivain, c'est toi. Moi, je ne suis que la façade. À toi la parole. 
M. Z. : OK...

M. W. : Mais d'abord, je te présente: tu te nommes Marc Zaffran, tu es né en 1955 à Alger. Tu as grandi en France à partir de 1962, tu as fait des études de médecine, puis pratiqué la médecine de famille à la campagne et dans un hôpital de province entre 1983 et 2008. Tu t'es installé à Montréal en 2009 et tu es résident permanent du Canada depuis 2011. Après ton premier roman, La Vacation, chez P.O.L en 1989, sous le pseudonyme de Martin Winckler — c'est-à-dire : bibi —, tu en as publié quinze autres, ainsi que des nouvelles, des contes, des essais sur la culture populaire - les téléséries en particulier - mais aussi sur le cinéma et la bande dessinée américaine, des manuels médicaux pour le grand public, des livres plus ou moins pamphlétaires consacrés à la relation de soin... En tout, une cinquantaine de bouquins. C'est ça ?

M. Z. : À peu près. Je n'ai pas fait le compte. Il y en a un ou deux que je préfère oublier.

M. W. : Vraiment?

M. Z. : Vraiment.

M. W. : Alors on n'en parlera pas. Mais on reparlera de la bibliorrhée.

M. Z. : Si tu veux.

M. W. : Commençons par une question simple qu'on te pose souvent, et qu'on se pose pour tous les écrivains : depuis quand écris-tu?

M. Z. : Je ne me rappelle pas exactement. Mes archives les plus anciennes sont des cahiers de nouvelles transcrites à l'âge de treize ans, mais probablement écrites au cours des deux années 
précédentes. À quatorze ans, je me mets à tenir un journal. Mais je ne sais pas s'il y a eu un «début », à proprement parler... Je vois ça comme une sorte de glissement insensible de la lecture à l'écriture, l'une dans le prolongement de l'autre. À l'époque, je passe mon temps à relire - donc, à réinventer - les livres que j'aime. Aujourd'hui, quand j'écris, j'ai le sentiment d'inventer — j'utilise ce mot parce qu'il signifie aussi bien "découvrir» qu'«imaginer» - le texte de chacun de mes livres à mesure que je l'écris afin de pouvoir enfin le lire.

M. W. : Pourquoi « enfin»?

M. Z. : Parce qu'une fois que j'en ai eu l'idée, mais tant que je ne suis pas assis pour l'écrire, il est là, quelque part, dans ma tête. Je m'en fais une idée fantomatique, souvent écrasante et impossible à circonscrire tout à fait. Quand je pense à un livre, quand l'idée m'en vient d'abord, j'ai le sentiment que je suis face à une sorte de cathédrale, un monde que je ne parviendrai pas à remplir. Et puis, une fois au travail, ça devient un monde à taille humaine, habité par les histoires. Je pense d'ailleurs que s'il me semble vide et vaste avant l'écriture, c'est précisément parce qu'il n'est pas encore habité. Par les personnages, par leurs histoires, par leurs paroles.

M. W. : Est-ce que le résultat écrit est décevant par rapport au «projet mental»?

M. Z. : Décevant, non. À mesure que le roman prend peu à peu ses contours, l'image initiale disparaît, et quand j'ai fini, je l'ai oubliée; le texte l'a remplacée. Une fois terminé, le roman est moins écrasant que l'idée initiale, mais aussi plus juste. Et j'espère, tout aussi riche pour le lecteur. Alors, déçu, non. Insatisfait, oui, parfois.

M. W. : Peux-tu préciser? 
M. W. : Il m'arrive, pendant l'écriture, et parfois après, de ne pas être content d'un chapitre, d'un passage, d'un tournant du livre, mais toujours pendant l'écriture et à ce moment-là je fais marche arrière, ou je coupe le texte qui ne « va nulle part » et je reprends. Mais je ne m'aventure jamais dans une direction qui ne me va pas, car si ça n'est pas la bonne, je cale très vite. Et puis je suis très exigeant avec moi-même quand j'écris pour P.O.L. Et Paul Otchakovsky-Laurens est plus exigeant que mes autres éditeurs... même si paradoxalement, c'est lui qui me fait le moins de remarques formelles. Mais il m'a déjà refusé - à juste titre - deux livres. Et ce refus a confirmé mon sentiment plus ou moins précis que le livre n'était pas fait pour être publié. Mais, d'un point de vue général, je ne rends pas un livre à un éditeur si je ne suis pas content du résultat, si je n'ai pas atteint l'objectif que je m'étais fixé en commençant.

M. W. : Et cependant, tu n'as pas tout le livre en tête avant de te mettre à le rédiger.

M. Z. : Non. J'ai le début et la fin, le plus souvent: j'écris beaucoup le début de mes romans, parfois plusieurs fois ; et je sais à peu près où je veux finir - sinon les dernières phrases, du moins la dernière scène ou la dernière situation. Une fois que j'ai mon début bien en main, je me lance. Il y a bien sûr, en cours d'écriture, des moments de tâtonnements, de doute, de réflexion intense sur la manière dont je vais «boucler » toutes les histoires de manière satisfaisante - à mes yeux, du moins -, mais comme je sais où je veux aller, je trouve toujours un moyen d'avancer.

M. W. : Et qu'est-ce qui vient en premier ? La narration ou le travail de la langue?

M. Z. : La narration, sans aucun doute. Le travail de la langue merci de ne pas utiliser le mot «style » - vient après. Il sert la narration. Je me relis et me réécris beaucoup, parce qu'il 
m'arrive souvent d'utiliser une tournure pour une autre, ou de me répéter lors du premier jet «au kilomètre », mais je n'écris jamais pour faire de belles phrases. J'essaie d'être aussi «efficace» que possible. Cela dit, j'aime les formules, les aphorismes, les métaphores, les bons mots, les phrases lapidaires... Je travaille particulièrement les dialogues, que je veux secs, rapides, incisifs. Et parfois décisifs.

M. W. : Plus tôt tu disais : « J'invente à mesure que j'écris ».

M. Z. : L'image la plus appropriée à mes yeux est celle que donnait Perec - et qui venait de Queneau, je crois : «L'écrivain est un rat qui construit le labyrinthe dont il se propose de sortir. » J'aime cette métaphore parce qu'elle contient beaucoup d'images entrelacées: le rat, animal intelligent qui renvoie aussi, en français, à l'expression " rat de bibliothèque » - et j'en suis un ; l'idée que l'écrivain explore son livre pour pouvoir le proposer - qu'il le conçoit, littéralement, en même temps qu'il le compose; l'image du labyrinthe, qui évoque aussi les striations du cortex, à la surface du cerveau, et que Perec transfère magistralement sur celle du puzzle de La Vie mode d'emploi, et des "cases à remplir» de ses Mots croisés ou d'Espèces d'espaces. Tous ces jeux sont faits à la fois pour "perdre » l'individu penché sur le puzzle ou le problème de mots croisés, et bien sûr, le lecteur ; mais ils présentent aussi les clés qui permettent de les résoudre, de les comprendre. Un jour, dans un château de la Loire, je suis entré dans un labyrinthe de verdure dont les sections étaient fermées par des portes électroniques. Il fallait découvrir une énigme et taper un code alphanumérique pour les franchir et le parcourir jusqu'au bout. À la fin, le visiteur accédait à une tour surplombant le labyrinthe et pouvait le contempler dans son ensemble. Quand j'écris un roman, c'est mon principal souci et la principale difficulté : construire une narration qui prenne tout son sens ou soit remise en perspective - à la dernière page. C'est vrai pour tous mes romans P.O.L, en tout cas. Je pense que c'est pour 
cette raison que La Vie mode d'emploi et $W$ ou le souvenir d'enfance m'ont autant marqué : c'est exactement le sentiment que j'ai eu en achevant ma lecture.

M. W. : Ce désir d'offrir au lecteur une perspective sur tout le livre, c'est une entreprise périlleuse...

M. Z. : Oui, parce que le risque encouru est celui d'échouer et, en quelque sorte, de trahir le lecteur qui m'a lu jusqu'au bout. C'est ce qui rend le projet exaltant, mais ça me met sous tension. Je me prépare au pire quand je suis prêt à envoyer le manuscrit, je me détends quand les membres de la maison d'édition m'en parlent, et je suis complètement rassuré quand des lecteurs inconnus m'écrivent: non seulement ils ont « compris » mais en plus, ils trouvent dans le livre des choses que je ne me rappelle pas avoir dites, et que je pense profondément. Cela dit, je n'ai pas, je pense, la même conception de la littérature que Perec. Je n'écris pas pour " encrypter » des sentiments ou des non-dits pénibles, comme il le fait dans toute son œuvre, et encore plus ouvertement dans ses poèmes lipogrammatiques, La Clôture, en particulier. J'écris, au contraire, pour mettre au jour ce qui, je le sens, est enfoui en moi, sans savoir toujours quoi. Et les sentiments que me renvoient les lecteurs me donnent à penser que j'y parviens, le plus souvent.

M. W. : Même dans les livres de «non-fiction »?

M. Z. : Pas tout à fait; les autres livres, qui sont les plus nombreux, sont plutôt voués à partager, à donner à voir et à savoir. Mais dans ce partage, il y a des valeurs et des sentiments, bien entendu.

M. W. : Mais ce n'est pas la même écriture, le même projet. 
M. Z. : Non. Écrire un livre sur la contraception, ou sur les téléséries, ou sur la relation de soins, c'est mettre sur le papier des notions, des informations, des idées, des opinions que je suis capable d'exprimer oralement. C'est garder la trace des paroles. Écrire un roman, c'est puiser dans, élaborer à partir d'un matériau plus inconscient, plus flou: les émotions, les représentations. Pour moi, la différence entre fiction et nonfiction réside là.

M. W. : Veux-tu dire que tu ne saurais pas inventer des fictions orales?

M. Z. : Je ne suis pas sûr que je pourrais les improviser comme le font les conteurs autour d'un feu de camp. Je me demande d'ailleurs si les conteurs improvisent jamais vraiment. Ou s'ils élaborent à partir d'histoires déjà racontées, ou qu'ils ont entendues et rapportent, réinventent ou développent.

M. W. : Tu n'improvises jamais ?

M. Z. : Pas la fiction. J'ai trop peur de me perdre. Même quand je faisais mes chroniques «improvisées » sur ARTE Radio, j'en écrivais une esquisse au préalable. Je développais au micro un texte parfois schématique, parfois assez élaboré. Je les ai réécrites ensuite ${ }^{1}$. Je faisais de même quand je tenais une chronique sur France Inter (la principale radio publique française) en 2002-2003, pour des raisons de durée, mais j'étais de toute manière plus confortable avec un texte déjà écrit.

\footnotetext{
${ }^{1}$ www.arteradio.com. Toutes ces chroniques, enregistrées sur trois ans, sont en ligne. Pour les retrouver rapidement dans le beau fouillis du site, taper « winckler» dans la zone de recherche. La première série a été reprise dans J'ai mal là (Les Petits matins, 2007), les deux suivantes dans Histoires en l'air (P.O.L, 2008).
} 
M. W. : La voix, c'est une constante, dans ce que tu écris. Mais tu ne t'en es pas rendu compte tout de suite, je crois...

M. Z. : Effectivement. Il y a dans tous mes livres beaucoup de dialogues, beaucoup de monologues intérieurs, beaucoup de narrations par les personnages, et quand je repense à mes tout premiers textes - y compris à mon « roman du grand tout " inédit, Les Cahiers Mercœur -, il est certain que j'y cherchais ma voix. Mes voix. Et mon dernier livre, En souvenir d'André, qui a peu de dialogues, est fait de monologues enchâssés. Il commence même par une « adresse » : un narrateur inconnu, en apparence extérieur à l'histoire, parle à l'auditeur silencieux qui va écouter parler le narrateur central du roman, Emmanuel. Et je me rends compte, en disant ça, que ça semble compliqué alors qu'à la lecture, c'est très simple, et on l'oublie très vite, et tant mieux! J'aime raconter mes histoires à haute voix, mais pour le faire, je dois d'abord les écrire.

M. W. : Écrire des romans, c'est raconter ?

M. Z. : Oui. Écrire un roman, c'est raconter une histoire, une histoire pleine de personnages et d'événements, comme on m'en a raconté autrefois, comme on m'en raconte aujourd'hui. Et je crois que cette perception du roman est précisément ce qui m'a attiré vers la littérature populaire, surtout anglosaxonne...

M. W. : Attiré ? Est-ce que ces genres ne t'ont pas formé, plutôt?

M. Z. : Il y a dix ans, c'est ce que j'aurais dit. Aujourd'hui, j'en suis moins sûr. Ce que je lis sur le fonctionnement du cerveau me donne plutôt à penser que le mien est fait pour ce genre d'histoires.

M. W. : Tu penses que certaines personnes sont faites pour certaines histoires et pas pour d'autres? 
M. Z. : La perception par les sens et l'intégration des informations par le cerveau diffèrent d'une personne à une autre. Certains de nous sont daltoniens, d'autres n'entendent pas certains sons, d'autres encore ont une hypersensibilité à la chaleur ou au contraire une insensibilité à la douleur. Je ne veux pas m'étendre sur le sujet mais je pense que oui, je suis fait pour certains types d'histoires, pas pour d'autres. Et je les ai trouvées dans la littérature populaire. Comme les Anglo-Saxons ont une littérature plus perméable, plus transversale que la littérature française, je m'y suis retrouvé chez moi. Mais pour en revenir à ma perception du roman, j'ai le sentiment - mais c'est intuitif, je ne saurais pas le démontrer ou donner des arguments théoriques - qu'il y a fondamentalement deux postures quand on écrit un roman. D'une part, la posture détachée, impersonnelle, de l'écrivain démiurge, qui reste à distance et, d'autre part, la posture engagée du narrateur qui se place tout près, se rapproche intimement de ses personnages en écrivant à la deuxième personne - le «tu » de Un homme qui dort, ou celui que j'ai employé dans La Vacation et La Maladie de Sachs - ou à la première, comme le font les écrivains de récits d'énigme, justement, à commencer par Edgar Poe et, bien sûr, Conan Doyle, Agatha Christie et, en France, des écrivains comme Maurice Leblanc: il invente Arsène Lupin en réponse à Sherlock Holmes en allant un cran plus loin puisqu'il laisse entendre que le Watson de Lupin, c'est lui-même. Et à mes yeux, ça n'est pas juste un effet de style, une figure obligée dans l'évolution du roman, c'est un « engagement narratif » que je rencontre surtout, à l'adolescence, dans le roman d'énigme et la science-fiction et non dans le roman français classique qu'on m'a fait étudier - Balzac, Flaubert, Stendhal - et qui m'a profondément ennuyé en classe, tant je l'ai trouvé lointain de ma sensibilité, de mes préoccupations. Alors que Wells, par exemple - Les Premiers Hommes dans la lune, La Guerre des mondes - me transporte. Et je frémis de joie quand je lis Le Monde perdu de Conan Doyle, dont le narrateur est un jeune journaliste, Malone. Dans tous ces livres, j'ai le sentiment que 
l'auteur me parle à l'oreille. C'est comme ça que je lis Jules Verne ou, même si ça peut paraître bizarre, le Victor Hugo de La Légende des siècles. De la poésie épique, grandiloquente, avec un souffle pas possible, une poésie qui ne peut qu'être dite à haute voix, devant une salle comble. Ou lue à ses enfants - je l'ai fait. Et lorsque, en 2004-2005, un éditeur de livres parlés, De Vive Voix, m'a proposé d'écrire des contes pour enfants, je les ai lus moi-même. Et mes plus jeunes s'endormaient en les écoutant.

M. W. : Cette "voix», tu l'entends donc davantage dans la littérature anglo-saxonne?

M. Z. : Disons que j'ai appris à l'entendre beaucoup plus tard chez des écrivains français, surtout ceux que publie P.O.L, car j'en lis peu d'autres, mais aussi chez les écrivains québécois que je n'ai pas encore assez lus. Pendant l'hiver 2011-2012, j'ai eu l'occasion de lire les états successifs du deuxième roman en travail d'un jeune écrivain qui vit à Québec, Richard Dallaire. Il s'intitulait Les Peaux cassées. À l'heure où je parle, il n'a pas encore été publié, mais ce qui m'a frappé, c'est cet engagement dans une narration extrêmement personnelle, physique, quand je la compare à celle d'écrivains français qui m'ont l'air hautain lorsqu'ils écrivent à la troisième personne et désabusés du monde quand ils disent « je ». On m'a suggéré que je ne les lisais pas bien, ou que je ne lisais pas les meilleurs, comme si on pouvait me convaincre que Bach est plus musical que Gershwin. Mais j'approche de la soixantaine et je sais que ce n'est pas une question de musicalité. C'est juste que Gershwin et Cole Porter me parlent, Bach et Mozart beaucoup moins. La littérature, c'est pareil. Si j'ai du plaisir immédiatement quand je commence un roman de John Irving ou Middlesex de Jeffrey Eugenides, ou Je compte les morts, de Geneviève Lefebvre, une Québécoise, ce n'est pas par snobisme ou par anti-snobisme, c'est parce que je me sens chez moi. Pas quand je lis Houellebecq ou Amélie 
Nothomb. Ni Proust, d'ailleurs - il me faut faire des efforts pour le lire. Ça veut bien dire que je ne suis pas fait pour lui.

M. W. : Mais il y a Perec.

M. Z. : Oui, il y a Perec et avant de le lire, j'avais dévoré Boris Vian et Paul Féval et Robert Merle et Stanislas-André Steeman et Sébastien Japrisot et, plus récemment j'ai lu Daniel Zimmermann, Claude Pujade-Renaud, René Belletto, Camille Laurens, Marie Darrieussecq. Et ce qui me touche, c'est la voix que j'entends en les lisant. Une voix qui monte aussi des textes de non-fiction, d'anthropologie, de psychologie évolutionniste, de vulgarisation scientifique que je lis en anglais. Encore une fois, ce n'est qu'un sentiment, mais ce qui me plaît, c'est sentir que le texte m'est raconté. C'est peut-être pour ça que lorsqu'un livre me tombe des mains, c'est parce que je le trouve trop écrit, trop pensé. Pas assez parlé. Les livres qui me touchent sont ceux que j'ai le sentiment d'entendre.

M. W. : Tu dis « les livres », pas « les auteurs».

M. Z. : Oui, lorsque j'aime un livre, je scrute les rayons des librairies pour lire tous les $\mathrm{Du}$ même auteur, et parfois je suis comblé. Parfois non. Ça dépend vraiment des écrivains et de ce qu'ils recherchent à chaque nouveau livre. Parfois, au bout de deux ou trois romans, leur voix change. En tout cas, c'est mon sentiment.

M. W. : Justement, les livres, comment les choisis-tu ?

M. Z. : Je ne lisais pas les critiques quand je vivais en France. J'allais dans les deux ou trois librairies du Mans, et dans quelques librairies à Paris quand j'y étais et je faisais le tour des tables, je prenais les livres entre mes mains, je les choisissais intuitivement, parfois après avoir longtemps tourné autour. Depuis que je vis à Montréal, je lis beaucoup les critiques anglo- 
saxons, du New York Times, de The Kirkus Review et d'un site Internet qui parle souvent de livres de sciences humaines : Arts \& Letters Daily. Le professionnalisme des critiques, leur absence de complaisance, leur humilité m'impressionnent et ils me font découvrir des bouquins incroyables. Je fréquente moins souvent les librairies, mais tout de même encore régulièrement. Olivieri sur Côte-des-Neiges, Paragraphe près de McGill et aussi Indigo et Chapters sur Sainte-Catherine. Et ces derniers temps, j'achète la plupart de mes livres au format électronique, moins encombrant, et j'en télécharge des dizaines. Je suis fasciné par la richesse de tout ce qui est à présent disponible dans le domaine public. J'ai également pris un abonnement annuel au site de François Bon, publie.net. Et je me mets à lire des classiques en anglais: Dickens, Melville, Poe, Conan Doyle à nouveau. Avec ma liseuse et ma tablette - j'ai les deux —, j'ai le sentiment de me balader avec une bibliothèque dans la poche. C'est réconfortant.

M. W. : Plus qu'écrire?

M. Z. : Plus souvent réconfortant. Je peux toujours lire, même quand je n'ai pas envie ou la force d'écrire. Avec la liseuse, je peux choisir le livre qui convient à mon humeur du moment, ou poursuivre n'importe où la lecture du gros bouquin que je ne veux pas lâcher. Avant la liseuse, quand je partais en voyage, je trimballais toujours avec moi plusieurs bouquins que je n'ouvrais pas. Mais il me les fallait. Au cas où. À présent, j'en emporte deux cents cinquante, mais ils ne pèsent rien.

M. W. : Et pourtant, les livres ont un poids dans ta vie...

M. Z. : On peut dire ça... J'ai vu mes enfants traverser leur enfance et parfois entrer dans l'adolescence avec des objets transitionnels - un poisson en peluche, des petits chiens, des Totoro -, mais je n'ai jamais eu d'ours en peluche. Les livres l'ont remplacé. L'autre jour, dans le métro, un de mes fils me 
demandait s'il y avait des romans que j'aurais voulu écrire. Il y en a plein. La liste était longue et, bien sûr, il y en avait beaucoup qu'il ne connaissait pas. J'ai ajouté : «Et c'est pour ça que j'écris. Pour écrire les romans que j'aimerais lire. »

M. W. : Mais tu ne lis pas tes romans.

M. Z. : Non, je ne peux pas les lire. Je peux traquer les coquilles, les feuilleter, les regarder d'un peu plus loin, mais pas les lire. Le seul moment qui ressemble à la lecture, c'est le moment de l'écriture. Comme si le texte naissait de la page. Quand j'écrivais au bille ou au stylo-plume, j'avais l'illusion que le glissement de ma main faisait apparaître les mots. Et j'avais toujours le bord de la paume taché d'encre. À présent, j'écris presque exclusivement à l'ordinateur. J'ai appris à taper en 1972, sur des machines mécaniques. Alors ça fait longtemps que je tape à dix doigts sans regarder le clavier, et je vois le texte apparaître en même temps que je le pense, j'ai le sentiment que ce sont les mots qui appellent mes pensées et non l'inverse. Que je pense le texte au moment où il se forme, pas avant. Et les neurobiologistes semblent dire que c'est le cas : quand nous faisons un geste, quand nous disons quelque chose, nous l'avons déjà décidé une fraction de seconde auparavant. Il y a un léger décalage entre la décision cérébrale et la conscience. Un décalage si court qu'on n'en a aucune conscience: il faut examiner le cerveau sous un appareil d'imagerie magnétique pour le mesurer. Ce qui signifie qu'écrire un roman, c'est, littéralement, porter la pensée à la conscience de celui qui écrit.

M. W. : En quoi est-ce plus vrai pour le roman que pour l'essai ou le manuel pratique - puisque tu en écris aussi ?

M. Z. : Là encore, c'est une perception personnelle - peut-être une illusion. Lorsque j'écris un essai ou un manuel - qui ont pour but d'exposer et de partager des opinions ou un savoir -, je puise dans des idées depuis longtemps énoncées 
verbalement, discutées, ressassées, reformulées, modulées par les échanges avec d'autres. Quand j'écris un roman, je puise dans un matériau peu ou pas abordé de vive voix. Intime, impalpable. Un mélange de souvenirs, de fantasmes personnels, de constructions inspirées par les histoires que j'ai lues ou entendues. Je pars d'une situation ou d'un cadre, je donne souvent à l'histoire des repères temporels, et je pars explorer tout ça. Je ne fais pas de plan, ou alors mon plan change au cours de l'écriture. C'est seulement une série de balises, comme des étapes grossièrement entourées sur une carte. Et, comme la carte n'est pas le territoire, je suis prêt, chemin faisant, à changer d'itinéraire. Pour l'essai ou le manuel, il en va autrement : j'ai besoin de faire un plan assez complet, parce que ce n'est pas une exploration, mais un inventaire : je cherche à faire un tour aussi complet que possible de ce que j'ai à dire sur le sujet. Et je crois qu'écrire des essais et des manuels m'a aidé à accepter qu'un roman, ça n'a pas besoin d'être exhaustif. Ça n'a pas pour vocation de l'être. Même s'il fait six cents pages... Prends Le Chœur des femmes, par exemple. Je ne cherchais pas à dresser un catalogue de tout ce que les femmes vivent et racontent aux médecins, je voulais seulement transformer mon expérience - ce que j'avais vu, entendu, compris - en une sorte d'oratorio romanesque. Certaines lectrices me disent: «Vous ne parlez pas de telle ou telle situation», comme si je pouvais à moi seul témoigner de toute l'expérience féminine. Aucun roman, aucun livre ne peut prétendre être la somme de tout ce que les humains ont à vivre. Mais comme dans $L a$ Maladie de Sachs, qui décrivait les relations entre un généraliste et la communauté qu'il soigne, ou Les Trois Médecins, qui transpose Dumas pour raconter les études de médecine d'un quatuor d'amis, je voulais décrire une expérience complexe, multiple. La polyphonie me l'a permis, et je suis encore un peu surpris d'y être parvenu. Mais je ne cherchais pas à être exhaustif. Ces lectrices pourraient écrire les chapitres du Chœur des femmes qui leur manquent, ajouter leur voix à celles des autres personnages. Dans mes romans, il y a toujours de la 
place pour l'expérience et l'imaginaire des lecteurs. Car j'attends d'un roman qu'il me laisse l'habiter et je ne cesse pas d'être lecteur quand j'écris.

M. W. : La polyphonie, tu l'as abandonnée pour écrire En souvenir d'André...

M. Z. : Pas abandonnée... Utilisée autrement. Dans les trois "gros» romans précédents, les voix s'élèvent à plusieurs reprises, et se répondent, comme en canon. Dans En souvenir d'André, c'est une polyphonie séquentielle, faite de récits consécutifs tissés dans la narration du personnage principal. Et le sujet s'y prêtait, puisque c'est l'ultime récit d'un homme qui a lui-même recueilli un grand nombre d'ultimes récits.

M. W. : C'était nécessaire ? Expérimental ?

M. Z. : Les deux, mon capitaine. Je voulais me prouver à moimême que j'étais capable de dire beaucoup en peu de pages, avec peu de personnages. Et je cherchais à produire à la lecture ce que pourrait être l'expérience des « veillées » décrites dans le livre.

M. W. : L'accompagnement de patients que l'on aide à mourir...

M. Z. : Oui, mais à présent, le livre est publié et plus le temps passe, plus j'ai le sentiment que le sujet central du livre n'est pas le suicide assisté, ni l'assistance à la fin de vie, mais la tentative, peut-être toujours vaine, de transmettre notre expérience émotionnelle. L'idée qui m'habitait pendant tout le temps que j'ai passé à l'écrire, c'est la fragilité de la transmission orale. Je reconnais que c'est paradoxal, puisqu'il s'agit d'un roman, forme beaucoup plus durable que les confidences recueillies dans l'instant.

M. W. : Tu écris pour conserver les paroles? 
M. Z. : Conserver, c'est momifier. J'écris pour transmettre ; en focalisant, en amplifiant, en affinant. C'est un peu un travail d'ingénieur du son, de mixage - tel que je l'imagine, car je n'y connais pas grand-chose. Les paroles - je n'écris que des paroles, je crois - que j'ai... enregistrées, puis retravaillées dans ma tête sont filtrées, modulées, gravées sur les pages et j'espère que non seulement le lecteur va les « entendre » mais qu'il les dira à son tour. Comme les chansons de Brassens ou de Brel ou de Ferré ou de Vian que je connais par cœur depuis toujours. Comme les voix des livres-disques que j'écoutais enfant, L'Île au trésor, Le Petit Prince, Le Livre de la jungle, La Marque jaune. Comme les sketchs d'humoristes, les poèmes de Rimbaud et d'Hugo que je connaissais par cœur. Comme les répliques de films... Je serais vraiment heureux que quelquesunes de mes phrases deviennent des signes de reconnaissance, de complicité. Des liens.

M. W. : Les trente-cinq bouquins publiés entre 1998 et 2000 , tu les as tous écrits pour transmettre?

M. Z. : Oui, mais cette bibliorrhée est née de l'ivresse, du désir de conquête qu'a provoqués le succès... et de la peur de ne pas être pris au sérieux si je ne publiais pas à tour de bras. Après la publication de mon premier roman, La Vacation, un homme que j'estimais m'a dit, assez méchamment: «C'est bien, mais c'est au deuxième roman qu'on voit l'écrivain. » Quand j'ai publié La Maladie de Sachs, un autre, que j'estimais autant, m'a déclaré : "C'est bien, mais ton premier roman, c'était un grand livre. » Un autre écrivain-médecin a enfoncé le clou en disant à peu près : «C'est un roman autobiographique. Je doute qu'il ait autre chose à raconter. " Ces phrases me sont longtemps restées en tête, car je doutais de jamais être considéré comme un écrivain "légitime », j'avais le sentiment que mon succès et ma réputation étaient usurpés. Et qu'un jour, si je m'en tenais à écrire un roman tous les trois ans, les lecteurs se rendraient compte que je n'avais pas grand-chose à dire. Alors j'ai cherché 
à prouver le contraire. J'avais envie de publier des livres de genres très différents, personne ne m'a obligé. Mais j'aurais pu prendre mon temps...

M. W. : Tu le regrettes?

M. Z. : En dehors des deux exceptions auxquelles j'ai fait allusion tout à l'heure, non. D'accord, je n'ai pas écrit seulement des livres nécessaires, comme je me jurais de le faire avant de me mettre à écrire à temps plein. Pendant ces dix années, j'ai écrit et publié ce que je voulais écrire depuis toujours: des romans policiers, de la $\mathrm{SF}$, de la critique, des essais polémiques, des manuels... Et tous ces livres ont modelé mon expérience d'écriture, comme mes cahiers d'adolescent ou, aujourd'hui, les entrées de mon site Internet ${ }^{2}$ et de mon blog littéraire ${ }^{3}$.

M. W. : ... sur lequel tu écrivais, tout récemment : «Écrire n'est pas un don.»

M. Z. : Ni un don, ni une malédiction. L'écriture est un outil. Écrire des livres, c'est un travail.

M. W. : Et pourquoi tout ce travail?

M. Z. : Pour écrire des livres qui comptent. Qui marquent. Qui changent la vie. Des livres que leurs lecteurs auraient voulu écrire. Des livres qui font éclore en eux les émotions qu'ils ne parvenaient pas à libérer jusque-là. Des livres qui, je l'espère, les feront écrire à leur tour.

M. W. : Tu penses à tout ça quand tu écris?

\footnotetext{
2 Winckler's Webzine (www.martinwinckler.com)

${ }^{3}$ Chevaliers des touches (www.wincklersblog.blogspot.com)
} 
M. Z. : Non. J'y pense tant que je n'écris pas. Quand je plonge dans l'écriture d'un livre, je ne pense plus qu'au livre, j'écris au kilomètre, jusqu'à atteindre l'état que décrivent les coureurs de marathon, le moment où ils ne sentent plus la douleur, où ils planent. Quand j'écris, je ne sens plus mon corps. Je suis en apesanteur. J'avance sans effort. Et chaque émotion, chaque désir peuvent se dire.

Montréal, 20 janvier 2013 